\title{
Continuous versus Intermittent Tamsulosin Therapy in Men with Lower Urinary Tract Symptoms due to Benign Prostatic Hyperplasia: A Randomized Control Trial
}

Waheed SMS ${ }^{1}$, Islam MS², Rakib MA ${ }^{3}$, Chowdhury $\mathrm{MA}^{4}$, Alam $\mathrm{MS}^{5}$

DOI: https://doi.org/10.3329/jafmc.v15i1.48635

\section{Abstract}

Introduction: Benign prostatic hyperplasia (BPH) is the most common benign tumour in men. About $50 \%$ of men at the age of 50 years develop BPH and about half of them report to the doctors with some form of lower urinary tract symptoms (LUTS).

Objectives: To see the therapeutic effect of Tamsulosin on alternate day administration in comparison to daily dose for men who had been suffering from LUTS due to BPH.

Materials and Methods: This clinical trial was carried out at Combined Military Hospital, Dhaka in a consecutive fashion for about 18 months. A total of 152 patients were selected from outpatient department and after assessing them through inclusion and exclusion criteria and grouped randomly in three groups Group-A (50 patients), Group-B (50 patients) and Group C (52 patients); all the patients were treated with Tablet Tamsulosin $(0.4 \mathrm{mg})$ daily for first three months. Then next 15 months they were treated as; Group-A received Tablet Tamsulosin $(0.4 \mathrm{mg})$ daily, Group-B received Tablet Tamsulosin $(0.4 \mathrm{mg})$ every alternate day and Group $C$ received placebo Tablet (Folic acid $5 \mathrm{mg}$ ) daily. Data were collected at the beginning and end of 3rd month, 4th month, 7th month and 18th month of study, in the form of international prostate symptom score (IPSS), maximum urinary flow per second (Qmax) and post-voidal residue (PVR).

Results: This study showed that at the initial three month, IPSS score declined in all three groups, Qmax improved and PVR reduced in all groups. In next phase of study; in Group-A patient there was progressive and significant improvement of all parameters (IPSS, Qmax and PVR) at the end of study. In Group-B patient also showed similar result at the end of study. In Group-C patient showed statistically significant difference in outcome in relation to Group-A and Group-B i.e. more in IPSS, less in Qmax and increase in PVR.

Conclusion: With this study it is clearly evident that tamsulosin $(0.4 \mathrm{mg})$ therapy significantly improves symptoms of men suffering for LUTS due to BPH. And alternate day therapy having similar therapeutic outcome in relation to every day therapy.

Key-words: Lower urinary tract symptom, Benign prostatic hyperplasia, Tamsulosin.

\section{Introduction}

Benign prostatic hyperplasia (BPH) is the most common benign tumour in men ${ }^{1}$. About $50 \%$ of men at the age of 50 years develop $\mathrm{BPH}$ and about half of them report to the doctors with some form of lower urinary tract symptoms (LUTS)2. Previously held notions that the clinical symptoms of BPH are caused simply by a massrelated increase in urethral resistance are too simplistic. It is now clear that a significant portion of LUTS is the result of age-related detrusor dysfunction. Moreover, bladder outlet obstruction itself may induce a variety of neural alterations in the bladder that contribute to symptomatology ${ }^{3}$. Whatever the origin, it is clear that BPH and LUTS increase with advancing age and are becoming more common because of an overall aging population. Watchful waiting, medical treatment and surgery are the modalities of treatment. But frequently the patients need at least some form of medical treatment for LUTS. Alpha (a)-adrenoreceptors blockers continue to be prominent form of medical therapy for these problems. It targets the dynamic component of BPH. Other forms of medical therapy i.e. 5 a-reductase inhibitors decrease the prostate mass but it require long time. Moreover, there appears to be no clear relationship between size of the prostate and severity of symptoms $s^{4,5}$. For these reason a-blockers are the main stay of medical treatment for LUTS due to BPH. The median probability $^{6}$ for symptomatic improvement with a-blocker therapy was estimated to be $74 \%$. Evidence indicates that apoptosis occurs in both stromal smooth muscle and glandular epithelial cells during treatment with a-blockers, achieving maximal effect approximately 6 months after initiation of therapy?.

But a1-adrenoreceptors are present not only in the smooth muscle of the lower urinary tract but also in the vascular bed of cardiovascular (CVS) and other parts of the body ${ }^{8}$. So even after using selective a -blocker (e.g. tamsulosin hydrochloride) some bothersome side effects are also observed ${ }^{9}$. These are dizziness, postural hypotension, asthenia, nasal stuffiness, peripheral edema and sexual dysfunction ${ }^{10,11}$. Tamsulosin is also relatively costly drug in our perspective. Regardless of the agents used, the current literature advocate continued use of a-blocker for their effect to be maintained. However to-date there is few studies of the potential of intermittent dose of these agents for lower urinary tract symptoms. Kaplan et al determined the safety and efficacy of intermittent alfuzosin therapy and Yanardag $\mathrm{H}$. et al with similar study with tamsulosin ${ }^{12,13}$. They reported that

1. Brig Gen SM Shameem Waheed, MBBS, FCPS (Surgery), FCPS (Urology), Professor of Surgery, AFMC, Dhaka (E-mail: waheedshameem@gmail.com) 2. Brig Gen Md Shahidul Islam, MBBS, MCPS, FCPS (Surgery), FCPS (Urology), Chief Surgeon, CMH, Dhaka 3. Col Mohammad Abdur Rakib, MBBS, MCPS, FCPS (Surgery), FCPS (Urology), Classified Specialist in Surgery, CMH, Dhaka 4. Lt Col Md Ashif Chowdhury, MBBS, FCPS (Surgery), FCPS (Urology), MRCS, MRCP, Classified Specialist in Surgery, CMH, Dhaka 5. Lt Col Md Shafiul Alam, MBBS, MCPS, FCPS (Surgery), FCPS (Urology), Classified Specialist in Surgery, CMH, Dhaka. 
intermittent therapy with these drugs are having similar outcome in comparison to continued therapy ${ }^{13}$. As stated it is relatively expansive in our perspective and is not without side effect and many a time patient may complains of sexual dysfunction and other side effects. In a recent study in Turkey by Goktas et al, it is suggested that intermittent tamsulosin therapy may cause decreased incidence of ejaculatory dysfunction ${ }^{14}$. This study was carried out to evaluate the effectiveness of intermittent tamsulosin therapy in men with LUTS due to BPH.

\section{Materials and Methods}

This clinical trial was carried out at Combined Military Hospital, Dhaka in a consecutive fashion for about 18 months. A total of 152 patients having LUTS due to BPH were selected from outpatient department and after assessing them through inclusion criteria; international prostate symptom score (IPSS) score more than 10, maximum urinary flow per second (Qmax) less than 15 $\mathrm{ml} / \mathrm{s}$, post-voidal residue (PVR) lower than $200 \mathrm{ml}$, and exclusion criteria and grouped randomly in three groups Group-A (50 patients), Group-B (50 patients) and Group C (52 patients); all the patients were treated with Tablet Tamsulosin $(0.4 \mathrm{mg})$ daily for first three months. Then next 15 months they were treated as; Group-A received Tablet Tamsulosin $(0.4 \mathrm{mg})$ daily, Group-B received Tablet Tamsulosin $(0.4 \mathrm{mg})$ every alternate day and Group $C$ received placebo Tablet (Folic acid $5 \mathrm{mg}$ ) daily. Data were collected and preserved in prescribed data collection sheet at the beginning and end of 3rd month, 4th month, 7th month and 13th month of study, in the form of IPSS (by selected questionnaires), Qmax (from the result of Uroflometry) and PVR (from Ultrasonography). At the end of study data were analyzed using SPSS for windows 16.0. Results were expressed as mean $\pm S D$ and range where appropriate. Statistical difference between groups were calculated by student's unpaired't' and Mann Whitney Rank test as applicable and ' $p$ ' value less than 0.05 was considered as significant. Informed written consent from the patients was taken in every steps of the study. Relevant permission was sought from the appropriate authority.

\section{Results}

Patients' age range was $43-82$ years and mean $\pm S D$ of age was $59.2 \pm 9.9,59.9 \pm 9.8$ and $59.4 \pm 8.1$ years in the Group-A, Group-B and Group- $C$ respectively and there was no statistical significant difference. Mean $\pm S D$ voided volume was $396 \pm 108,430 \pm 163$ and $379 \pm 147 \mathrm{ml}$ in the Group-A, Group-B and Group-C respectively and the difference not significant (Table-I). Mean $( \pm S D$ ) IPSS level of the three groups at the start at the study IPSS-B0 was 20.6 \pm 4.8 , $20.4 \pm 4.7$, and $20.1 \pm 4.3$ respectively and this difference among groups was not statistical significant ( $p>0.05$ ). Mean ISD IPSS level after three months of initial treatment IPSS-B1 was $17.4 \pm 4.1$, $17.9 \pm 4.2$ and $17.0 \pm 2.3$ respectively in the three groups which was statistically not significant ( $p>0.05)$ among the groups. The IPSS-B1 level was, however, significantly lower compared to their corresponding IPSS BO level $(\mathrm{p}<0.001)$ (Table II).

Mean \pm SD level at first follow-up (IPSS-F1); was 15.8 \pm 4.0 , $16.5 \pm 4.2$ and $17.6 \pm 4.0$ respectively in the three groups. IPSS-F1 level in Group-A did not show statistically significant difference compared to Group-B ( $p>0.05)$. The IPSS-F1 value in Group-C was significantly higher compared to Group-A $(p<0.05)$, but no significant difference was observed compared to Group-B $(p>0.05)$. At the second follow-up mean $\pm S D$ IPSS-F2 level was $14.6 \pm 4.0,15.4 \pm 4.7$, and $20.1 \pm 4.6$ respectively in the three groups. The IPSS-F2 level between Group-A and Group-B did not show any statistically significant difference ( $p>0.05)$. The IPSS-F2 level was significantly higher in Group-C compared to Group-A and Group-B ( $p<0.001$ for both). At third follow-up mean \pm SD IPSS-F3 level was $13.9 \pm 3.8,14.3 \pm 4.5$, and $22.7 \pm 5.0$, in the three groups respectively. The mean $\pm S D$ value in the Group-A and Group-B did not show any significant difference $(p>0.05)$. The mean $\pm S D$ in the Group-C was significantly higher compared to Group-A and Group-B ( $p<0.001)$ for both. Mean \pm SD IPSS-F1, IPSS-F2 and IPSS-F3 in follow-up periods showed gradual decline in both Group-A and Group-B. The fall was significantly lower in both groups in the follow up periods (IPSS-F1, IPSS-F2 and IPSS-F3) compared to IPSS-B1 and also among the follow up periods $(p<0.001)$. The mean IPSS levels in the different visits was also shown in the figure-1 which had shown gradual fall in IPSS level in the Group-A and Group-B but rise in Group-C (Figure-1). In the Group-C IPSS levels in the three follow up periods (IPSS-F1, IPSS-F2 and IPSS-F3) showed an upward trend and had highest in third follow up (IPSS-F3). IPSS-F1 level did not show any statistically significant difference compared to IPSS-B1 $(p>0.05)$, IPSS-F2 and IPSS-F3 value was significantly higher compared to IPSS-B1 $(p<0.001)$ for both and between the follow-up $(p<0.001)$ periods (Table-II).

Mean $\pm S D$ Qmax at the begining of the study (Qmax-B0) in the three groups was $11.7 \pm 1.8,11.5 \pm 2.1$, and $11.3 \pm 2.5$ in the Group-A, Group-B, and Group-C respectively. No significant difference was observed regarding Qmax-B0 among the three groups (TableIV). Mean $\pm S D$ Qmax-B1 value after initial treatment for three months was $12.3 \pm 1.3,12.4 \pm 1.6$, and $12.8 \pm 1.7$ respectively. No significant difference was observed among the groups $(p>0.05)$. At the first follow up mean $\pm S D$ Qmax value was $12.8 \pm 1.2$, $12.8 \pm 1.0$, and $12.2 \pm 2.2$ in the three groups respectively. Qmax-F1 value in Group-A and Group-B did not show statistical significant difference ( $p>0.05$ ). Mean $\pm S D$ Qmax-F1 value in Group-C had significantly lower value compared to Group-A $(p<0.05)$ but no difference was observed compared to Group-B ( $p>0.05)$. At the second follow up mean $\pm S D$ Qmax-F2 value was $13.3 \pm 1.1$,

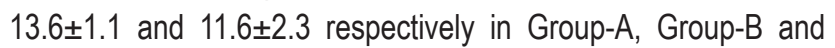
Group-C. Mean $\pm S D$ Qmax-F2 value in Group-A and Group-B did not show any significant difference ( $p>0.05)$. Mean $\pm S D$ Qmax-F2 value in Group $C$ was significantly lower compared to both Group-A and Group-B $(p<0.001)$ for both. At the final follow up mean $\pm S D$ Qmax-F3 value was $13.7 \pm 1.1,13.9 \pm 1.2$ and $10.9 \pm 2.3$ respectively in Group-A, Group-B and Group-C respectively. Mean $\pm S D$ value of $Q m a x-F 3$ between Group-A and Group-B did not show any significant difference ( $p>0.05)$. Mean $\pm S D$ Qmax-F3 value in Group-C was significantly different compared to Group-A and Group-B $(p<0.001)$ for both. The mean Qmax levels in the different visits was also shown in the Figure-2 which had shown steady state rise of the level in Group-A and Group-B but gradual fall in the placebo Group-C. Qmax value was compared between 
visits. Mean $\pm S D$ value between $Q \max -\mathrm{B} 1$ and $\mathrm{Qmax}-\mathrm{F} 1$ did not significantly different ( $p>0.05)$. Qmax value in the first (Qmax-F1) and second follow up (Qmax-F2) was significantly higher compared to Qmax-B1 ( $p<0.001)$ for both. Mean $\pm S D$ Qmax-F2 and Qmax-F3 was also significantly different compared to Qmax-F1 ( $p<0.01$ and $p<0.001)$ respectively. Mean $\pm S D$ Qmax-F2 and Qmax-F3 did not show any significant $(p>0.05)$ difference (Table-III).

Postvoidal residue median (range) in $\mathrm{ml}$ at the beginning of the study (PVR-B0) was 89(0-190), 90(13-160) and 97(18-190) in Group-A, Group-B and Group-C respectively. PVR level in the three different groups did not show any statistical difference $(p>0.05)$. At the end of initial treatment of three months, PVR-B1 was 65(0-146), 50(0-146) and 53(0-116) in the Group-A, Group-B and Group-C respectively. PVR-B1 level in the three groups also did not show significant difference among the groups $(p>0.05)$. In the first follow up PVR-F1 level was 55(0-92), 45(0-140) and 62(10-187) in the Group-A, Group-B and Group-C respectively. PVR-F1 level in the three groups did not show significant statistical difference $(p>0.05)$. In the second follow up PVR-F2 was 45(0-94), 46(0-134) and 87(13-193) in the Group-A, Group-B and Group-C respectively. PVR-F2 level in the Group-A and Group-B did not show any statistical significant difference ( $p>0.05)$. PVR-F2 level in Group-C was significantly higher compared to group-A and Group-B $(p<0.001)$ for both. In the final follow up period PVR-F3 was $40(0-88), 42(0-130)$ and 112(17-199) in the Group-A, Group-B and Group-C respectively, PVR-F3 level in the Group-A and Group-B did not show any significant difference $(p>0.05)$. PVR-F3 level in Group-C was significantly higher compared to Group-A and Group-B ( $p<0.001)$ for both. PVR median values $(\mathrm{ml})$ of the three groups were also shown in the Figure-3 which had shown almost similar response in the two treated groups and rise in median value of the Group-C. PVR median level of the three groups was compared within visits. Median PVR-B1 in the three groups was significantly lower compared to PVR-B0. Median PVR of the Group-A and Group-B in the subsequent visit showed continued downward trends in the subsequent follow up visits $(p<0.001)$. Only in Group-B no significant difference was observed between PVR-F1 and PVR-F2 levels (Table-IV). In the Group-C median PVR-B1 and in subsequent follow up visits showed upward trend and was significantly $(p<0.001)$ higher between visits (Table-IV).

Table-I: Age and voided urinary volume of the study subjects

\begin{tabular}{|c|c|c|}
\hline Groups & Age (years) & Voided volume (ml) \\
\hline Group-A(n=50) & $59.2 \pm 9.9$ & $396 \pm 108$ \\
\hline Group-B(n=50) & $59.9 \pm 9.8$ & $430 \pm 163$ \\
\hline Group-C(n=52) & $59.4 \pm 8.1$ & $379 \pm 147$ \\
\hline
\end{tabular}

Table-II: IPSS in the different follow-up of the study subjects

\begin{tabular}{|c|c|c|c|c|c|}
\hline Groups & IPSS-B0 & IPSS-B1 & IPSS-F1 & IPSS-F2 & IPSS-F3 \\
\hline Group- $\mathrm{A}(\mathrm{n}=\mathbf{5 0})$ & $20.6 \pm 4.8$ & $17.4 \pm 4.1$ & $15.8 \pm 4.0$ & $14.6 \pm 4.0$ & $13.9 \pm 3.8$ \\
\hline Group $\mathrm{B}(\mathrm{n}=\mathbf{5 0})$ & $20.4 \pm 4.7$ & $17.9 \pm 4.2$ & $16.5 \pm 4.2$ & $15.4 \pm 4.7$ & $14.3 \pm 4.5$ \\
\hline Group $\mathrm{C}(\mathrm{n}=\mathbf{5 2})$ & $20.1 \pm 4.3$ & $17.0 \pm 2.3$ & $17.6 \pm 4.0$ & $20.1 \pm 4.6^{*}$ & $22.7 \pm 5.0^{*}$ \\
\hline
\end{tabular}

Note: IPSS B1, IPSS at the end of initial three month; IPSS-F1:
IPSS at first follow up (at the end of 4th month); IPSS-F2, IPSS at second follow up (at the end of 7th month); IPSS-F3, at third follow up (at the end of 13th month). ${ }^{*}$ significantly $(p<0.001)$ different compared to Group-A and Group-B.

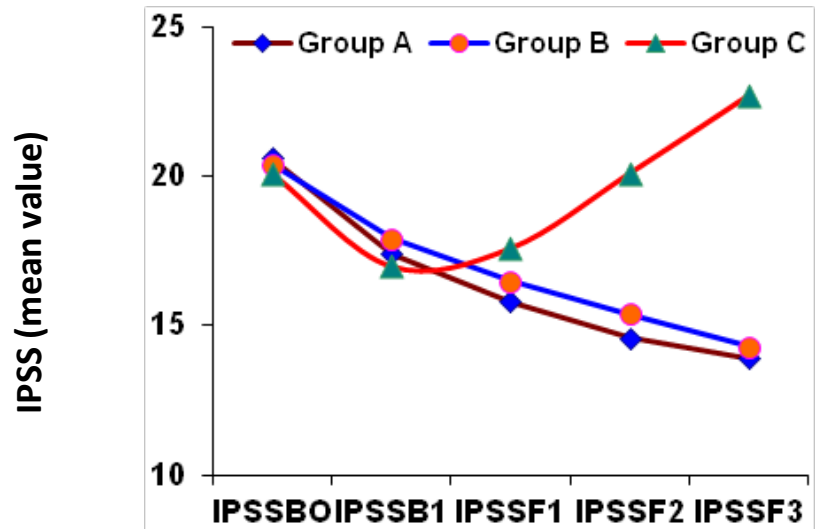

Figure-1: Mean IPSS value of the three study groups in different visits

Table-III: Maximum urine flow per second (Qmax) in the different follow-up visits of the study subjects

\begin{tabular}{|c|c|c|c|c|c|}
\hline Groups & Qmax-B0 & Qmax-B1 & Qmax-F1 & Qmax-F2 & Qmax-F3 \\
\hline Group-A & $11.7 \pm 1.8$ & $12.3 \pm 1.3$ & $12.8 \pm 1.2$ & $13.3 \pm 1.1$ & $13.7 \pm 1.1$ \\
\hline Group-B & $11.5 \pm 2.1$ & $12.4 \pm 1.6$ & $12.8 \pm 1.0$ & $13.6 \pm 1.1$ & $13.9 \pm 1.2$ \\
\hline Group-C & $11.3 \pm 2.5$ & $12.8 \pm 1.7$ & $12.2 \pm 2.2$ & $11.6 \pm 2.3^{*}$ & $10.9 \pm 2.3^{*}$ \\
\hline
\end{tabular}

Note: Results were expressed as mean $\pm S D$. Student's unpaired 't' test was performed to compare statistical difference between groups. *significantly $(p<0.001)$ different compared to Group A and Group B.

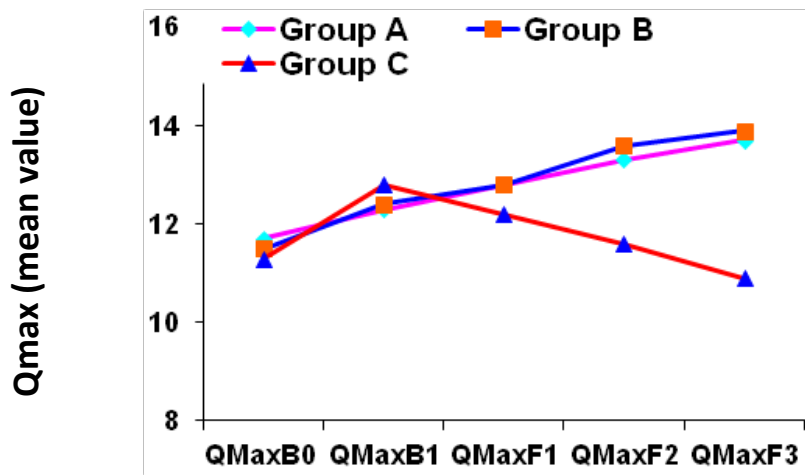

Figure-2: Mean $Q \max$ value of the three study groups in different visits

Table-IV: Postvoidal residue (PVR) in the different follow-up of the studysubjects

\begin{tabular}{|c|c|c|c|c|c|}
\hline Groups & PVR B0(ml) & PVR B1 (ml) & PVR 1 (ml) & PVR F2(ml) & PVR F3(ml) \\
\hline Group A & $89(0-190)$ & $65(0-146)$ & $55(0-92)$ & $45(0-94)$ & $40(0-88)$ \\
\hline Group B & $90(13-160)$ & $50(0-146)$ & $45(0-140)$ & $46(0-134)$ & $42(0-130)$ \\
\hline Group C & $97(18-190)$ & $53(0-116)$ & $62(10-187)$ & $87(13-193)^{*}$ & $112(17-199)^{*}$ \\
\hline
\end{tabular}

Note: Results were expressed as median (range). Mann Whitney Rank Sum test was performed to compare statistical difference between two groups. PVR-B1, PVR at the end of initial three month; PVR-F1: PVR at first follow up (at the end of 4th month); PVR-F2, 
PVR at second follow up (at the end of 7th month); PVR-F3, at third follow up (at the end of 13th month). * significantly $(p<0.001)$ different compared to Group A and Group B.

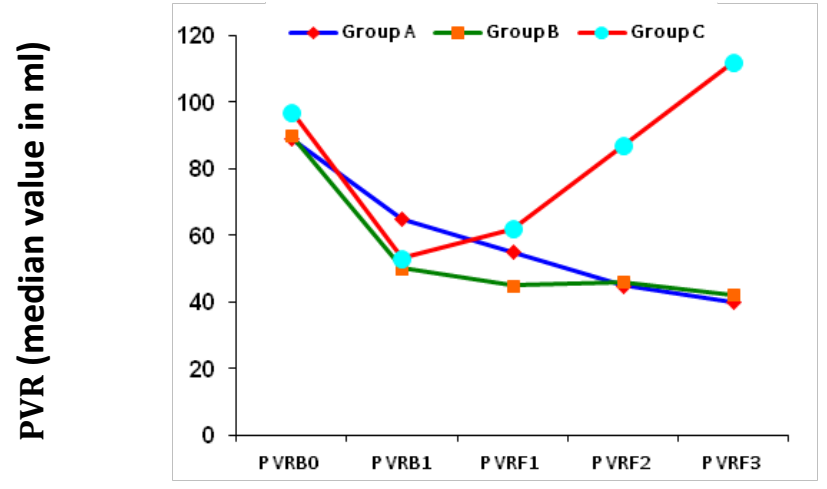

Figure-3: Median PVR level in milliliter of the three study groups in different visits

\section{Discussion}

Many treatment methods for LUTS due to BPH have been developed during recent years. Although studies showed that several invasive treatments considerably reduced the symptoms and increased the quality of life of BPH patients ${ }^{15,16}$, conservative therapies including observation and pharmacotherapy should be initially considered. Selective alpha 1-adrenoceptor antagonists, including tamsulosin, are often used for the treatment of symptoms of BPH all over the world. Three a1-adrenoceptors subtypes can be identified in pharmacological studies, namely $a 1 a, a 1 b$ and $a 1 d$. The a1a subtype predominates in the prostate capsule and it is responsible for mediating smooth muscle tone. Currently available a1-adrenoceptor antagonists, such as alfuzosin, terazosin prazosin and doxazosin, are not selective for any a1-adrenoceptor subtypes ${ }^{17}$. Tamsulosin is the first prostate selective a1-adrenoceptor antagonist that is 7 to 38 times more selective for the a1a-adrenoceptor subtype that is functional in the prostate than for the $\mathrm{a} 1 \mathrm{~b}$-adrenoceptor subtype that is functional in human blood vessels ${ }^{18}$. Compared with the other available a1adrenoceptor antagonists, tamsulosin has the clinical advantage that treatment can immediately be initiated with the full therapeutic dose $^{8}$. Nonsubtype selective a1-adrenoceptor antagonists must be initially administered upward until the therapeutic dose is achieved. This is required to decrease the occurrence of side effects, especially symptomatic postural hypotension after the first dose ${ }^{19}$. More importantly, even the $0.4 \mathrm{mg}$ dose was safely administered without dose titration ${ }^{20}$. Tamsulosin treatment of $\mathrm{BPH}$ patients for more than 12 months also showed a sustained stable efficacy ${ }^{21}$. On the other hand, intermittent tamsulosin therapy is also cost effective and receiving of $0.4 \mathrm{mg}$ once daily every other day is also simple for patients.

In this study men \pm SD IPSS at the start of study was similar in all the three groups. Three months Tamsulosin therapy caused significant improvement of symptoms. After three months of treatment, withdrawal of drug caused re-appearance of similar symptoms at the level of initial symptom at 7 th month and further deterioration of symptoms at 13th month. On the other hand, at the end of 13th month, symptoms remained almost similar in both the two groups where tamsulosin used continuously and intermittently. Similar result observed in case of Qmax. At the end of three months of tamsulosin therapy flow improved in all the three groups. Treatment with placebo showed gradual determination of flow and it come back to initial level prior treatment at 7 th month and further deteriorated at the end of 13th month. But in both the groups of continuous and intermittent tamsulosin therapy gradual improvement noticed in both the two groups almost in similar fashion. Lastly while evaluating PVR similar tendency noticed. At the end of initial three months therapy, PVR decreased significantly. But treatment with placebo showed gradual increase in PVR and on the other hand other two groups showed gradual decline in similar ways. So, from this study it is clearly evident that intermittent tamsulosin therapy for LUTS due to BPH is having similar outcome to that of continuous therapy (Insignificant difference is noticed statistically in outcome at the end of study).

This was an unpaid study, carried out in a single centre. Patients were consecutively selected. They were scrutinized in a common environment along with other types of patients. Most of the patients were from retired armed forced personnel living in relatively poor socioeconomic conditions far away from capital city. Indoor treatment at Armed Forces is free of cost and more over due to our cultural set up there is a general tendency to express symptoms in an exacerbated way which may not co-relate with findings of investigation. For that reason in this study population mean \pm SD IPSS showed around 20 marked as serve symptoms. But their PVR showed moderate obstruction. Again there is long queue in the Ultra sonogram department, where there is always a tendency to hurry. That may be a reason to show relatively high PVR in study subjects. Whatever may be the situation at the end of study it is clearly evident that tamsulosin definitely improves the state of LUTS of the study subjects and intermittent therapy of tamsulosin is having almost similar outcome to that of continuous tamsulosin therapy.

Dizziness and abnormal ejaculation (retrograde ejaculation, decreased volume or absent ejaculate) are the most common adverse events (4.5\%) associated with $0.4 \mathrm{mg}$ tamsulosin once daily with headache, asthenia, rhinitis, postural hypotension and palpitation seen less frequently ( $1 \%$ to $2 \%$ of patients) $)^{11}$. Except for dizziness and abnormal ejaculation adverse events show a similar incidence that of placebo ${ }^{11}$. This effect is probably related to a1blockade by tamsulosinat the bladder neck, in prostatic smooth muscle and the vas deferens, and it can be reversed by withdrawal of the drug ${ }^{12}$. Recently, a study in Turkey by Goktas et al showed that intermittent tamsulosin therapy may cause significant improvement of ejaculatory problem, where there is significantly less incidence of abnormal ejaculation ${ }^{16}$. The data in this study clearly indicate that patients who received tamsulosin daily did not far better than men who received tamsulosin every other day. The removal of active medication resulted in return to baseline of symptoms and the peak urinary flow rate at 3 and 6 months of randomization, and these values did not significantly differ from values at initiation. These results are similar to those in the study of Kaplan et al and 
Yadrang et al but "they differ from those of Debruyne who reported that BPH symptoms did not return to baseline after the withdrawal of terazosin ${ }^{13}$. Recently Yokoyam et al studied 75 patients with LUTS who are having relatively small volume prostate and good flow rate; they stopped a-blocker medication once their symptoms improved and 42 patients were able to maintain good condition without medication after 12 months ${ }^{14}$. A potential role of medical therapy is to prevent the development of BPH or its progression.

\section{Conclusion}

Multicenter, randomized, double-blind, placebo-controlled studies have unequivocally demonstrated the safety and efficacy of a blockers for the treatment of BPH. It was evident from this study that intermittent (on alternate day) therapy with Tamsulosin $(0.4 \mathrm{mg})$ for LUTS due to BPH showed a similar therapeutic efficacy to that of continuous therapy.

\section{References}

1. Tanagho EA, Mcannich JW. Smiths General Urology. Lange Medical Book. Mc. Graw -Hill USA 16th ed, 2004:367.

2. Salam MA. Principles and Practice of Urology. MAS publication, Dhaka, 2002:640.

3. Walsh PC, Retik AB, Vaughan ED, Wein AJ. Campbell's Urology. Saunders, 8th ed, 2002:1297-1378.

4. Sapiro E, Lepor H. Pathophysiology of clinical benign prostatic hyperplasia, Urol Clin North Am 1995; 22:285-90.

5. Wadie BS, Ibrahim EH, De La Rosetle JJ et al. the relationship of the International Prostate Symptom Score and objective parameters for diagnosing bladder out let obstruction. Part I; when statistics fail. J Urol 2001; 165:32-4.

6. Rossi C, Kortmann BB, Sonke GS et al. a-Blocked improves symptoms suggestive of bladder outlet obstruction but fails to relieve it. J Urol 2001; 165:38.

7. Crullar DC, Kyprianou N. Future concepts in the medical therapy of benign prostatic hyperplasia. Curr Opin Urol 2001; 11:27-33.

8. Schulman CC, Lock TM, Buzelin TW et al. Long term use of tamsulosin to treat lower urinary tract symptoms/benign prostatic hyperplasia. J Urol 2001; 166:1358.

9. Schulman CC, Corturiend J, Jones U et al. Tamsulosin: 3-year long term efficacy and safety in patients with lower urinary tract symptoms suggestive of benign prostatic obstruction: analysis of a European, multinational, multicenter, open label study. European tamsulosin study group .Eur Urol 1999; 36:609.

10. Dunn CJ, Matheson A, Faulds DM. Tamsulosin. A review of its pharmacology and therapeutic efficacy in the management of lower urinary tract symptoms. Drugs Aging 2002; 19:135.

11. Abrams P, Speakman M, Stott $M$ et al. A dose ranging study of the efficacy and safety of tamsulosin, the first prostate selective alpha 1 A-adrenoceptor, antagonist, in patients with benign prostatic obstruction (symptomatic benign prostatic hyperplasia). B J Urol 1997; 80:587.

12. Kaplan SA, Reis R, Cologna A et al. Intermittent alpha-blocker therapy in the treatment of men with lower urinary tract symptoms. Urology 1998; 52:12.

13. Yanardag H, Goktas S, Kabir Y. Intermittent tamsulosin therapy in men with lower urinary tract symptoms. J Urol 2005; 173:155-7.

14. Forray C, Brad JA, Wetzel JM et al. The a1 adrenergic receptor that mediates smooth muscle contraction in human prostate has the pharmacological properties of a cloned human a1C subtype. Mol Phamacol 1994; 45:703-8.

15. Shapiro E, Lepor H. Pathophysiology of clinical benign prostatic hyperplasia. Urol Clin North Am 1995; 22:285.

16. Algaba F. Pathophysiology of benign prosttic hyperplasia. Eur Urol 1994; 25(Suppl 1):3-5.

17. Schwinn DA. The role of alpha 1-adrenergic receptor subtypes in lower urinary tract symptoms. BJU Int 2001; 88(Suppl 2):27-34.

18. Walsh PC, Madden JD, Harrod MJ et al. Familial incomplete male pseudohermaphroditism, type 2. Decreased dihydrotestosterone formation in pseudovaginalperineoscrotal hypospadias. N Engl J Med 1974; $291: 944$.

19. Bartsch W, Becker H, Pinkenburg FA et al. Hormone blood levels and their inter-relationships in normal men and men with benign prostatic hyperplasia (BPH). Acta Endocrinol 1979; 90:727.

20. Caine M. Alpha-adrenergic blockers for the treatment of benign prostatic hyperplasia. Urol Clin North Am 1990; 17:641.

21. Roehrborn CG, McConnell JD, Lieber M et al. Serum prostate-specific antigen concentration is a powerful predictor of acute urinary retention and need for surgery in men with clinical benign prostatic hyperplasia. PLESS Study Group. Urology 1999; 53:473. 